\title{
IncRNA MAGI2-AS3 Exerts Antioncogenic Roles in Hepatocellular Carcinoma via Regulating the miR-519c-3p/TXNIP Axis
}

\author{
Huamei Wei, ${ }^{1}$ Qianli Tang, ${ }^{2}$ Anmin Wang, ${ }^{3}$ Ya Zhang, ${ }^{3}$ Zebang Qin, ${ }^{3}$ Wenchuan Li, ${ }^{2}$ \\ Zuoming $\mathrm{Xu},{ }^{2}$ Jianchu Wang, ${ }^{2}$ and Jian $\mathrm{Pu} \mathbb{D D}^{2}$ \\ ${ }^{1}$ Department of Pathology, Affiliated Hospital of Youjiang Medical University for Nationalities, Baise, Guangxi 533000, China \\ ${ }^{2}$ Department of Hepatobiliary Surgery, Affiliated Hospital of Youjiang Medical University for Nationalities, Baise, \\ Guangxi 533000, China \\ ${ }^{3}$ Graduate College, Youjiang Medical University for Nationalities, Baise, Guangxi 533000, China
}

Correspondence should be addressed to Jian Pu; yj_pujian@163.com

Received 21 February 2021; Accepted 13 August 2021; Published 27 August 2021

Academic Editor: Thomas R. Chauncey

Copyright ( 2021 Huamei Wei et al. This is an open access article distributed under the Creative Commons Attribution License, which permits unrestricted use, distribution, and reproduction in any medium, provided the original work is properly cited.

\begin{abstract}
Introduction. Our work was aimed to explore the mechanisms of MAGI2 antisense RNA 3 (MAGI2-AS3) in regulating hepatocellular carcinoma (HCC) carcinogenesis. Methods. MAGI2-AS3, microRNA-519c-3p (miR-519c-3p), and thioredoxin interacting protein (TXNIP) levels in HCC were detected by the RT-qPCR method. Cell proliferation and apoptosis rate were measured using Cell Counting Kit-8 assay and flow cytometry assay. Relationship between MAGI2-AS3, TXNIP, and miR-519c$3 p$ were analyzed via luciferase activity assay, RNA pull-down assay, and RNA immunoprecipitation assay. Mouse xenograft models of HCC were conducted to explore the roles of MAGI2-AS3 in vivo. Results. MAGI2-AS3 levels were elevated, and miR519c-3p decreased in HCC. MAGI2-AS3 overexpression inhibits while its knockdown stimulates HCC cell growth through miR519c-3p. Moreover, miR-519c-3p overexpression stimulates HCC cell growth. MAGI2-AS3 serves as competing endogenous RNA (ceRNA) of miR-519c-3p to regulate TXNIP in HCC. And, TXNIP upregulation weakened the influence of MAGI2-AS3 knockdown on HCC cell behaviors. Additionally, MAGI2-AS3 overexpression suppressed HCC tumor growth in vivo. Conclusion. MAGI2-AS3 inhibits HCC tumorigenesis through miR-519c-3p/TXNIP axis in vitro and in vivo, indicating MAGI2-AS3 plays a crucial role in HCC development.
\end{abstract}

\section{Introduction}

In recent years, the numbers of newly diagnosed and death cases for liver cancer have gradually increased and becomes a cancer type with highest mortality worldwide [1]. Although the improvements are on treatment methods, the prognosis of liver cancer remains poor due to most patients are diagnosed at advanced stages [2]. Hepatocellular carcinoma (HCC) accounts for 75-85\% of all liver cancer cases all over the world [3]. Hence, it is essential to discover molecular markers behind HCC development.

RNA can be generally divided into two groups, protein coding and noncoding RNAs, based on whether or not they have the ability to code proteins. Noncoding RNAs (ncRNAs) represents about $98 \%$ of all genome transcripts and can be classified into short ncRNAs ( $<200$ nucleotides) and long ncRNAs (lncRNA, $>200$ nucleotides) [4]. Although only some of these lncRNAs have been functionally characterized, they are found to contribute the malignant behaviors of cancer cells and therefore could not be merely regarded as transcriptional "noise" [5].

MAGI2 antisense RNA 3 (MAGI2-AS3) is a lncRNA with dual roles, tumor suppressive role and oncogenic role, in cancers. For instance, MAGI2-AS3 was a recently found decreased expression in HCC and could inhibit HCC cell growth and metastasis via recruiting KDM1A to enhance the H3K4me2 demethylation status at the promoter region of RACGAP1 [6]. On the contrary, MAGI2-AS3 was found elevated expression in colorectal cancer and drives cancer tumorigenesis in vitro and in vivo through regulating the 
miR-3163/TMEM106B axis [7]. However, the roles of MAGI2-AS3 in HCC remain to be further explored.

Mechanistically, lncRNA serves as competitive endogenous RNA (ceRNA) for miRNA to modulate messenger RNA (mRNA) expression [8]. In this work, we focused on miR-519c-3p which has been revealed that could contribute to HCC growth and metastasis in vitro and in vivo by targeting B cell translocation gene 3 [9]. However, the mechanisms regarding the upregulation status of miR-519c$3 p$ in HCC remain to be explored.

Here, we hypothesized MAGI2-AS3 may function as ceRNA for miR-519c-3p in HCC. The expression levels of MAGI2-AS3 and miR-519c-3p in HCC were analyzed. Moreover, gain and loss-of-function studies were performed to investigate the associated mechanisms of MAGI2-AS3 in HCC.

\section{Materials and Methods}

2.1. Patients Samples. HCC tumor tissues and adjacent noncancerous tissues were collected from 27 patients underwent treatment at Affiliated Hospital of Youjiang Medical University for Nationalities with a protocol approved by our hospital. These tissues were all stored at $-80^{\circ} \mathrm{C}$ refrigerator until usage. The written informed consent was obtained from all participants.

2.2. Cell Lines and Culture. Dulbecco's modified Eagle medium (DMEM) and 10\% fetal bovine serum (Invitrogen, Carlsbad, CA, USA) were used to incubate HCC cells (Huh7, Hep3B, SNU-182) and normal liver cell (THLE-3) obtained from ATCC. The incubation condition was maintained at $37^{\circ} \mathrm{C}$ with $5 \%$ of $\mathrm{CO}_{2}$.

2.3. Transfection. To overexpress MAGI2-AS3 or thioredoxin interacting protein (TXNIP), their full-length sequences were inserted into pcDNA3.1. To overexpress miR519c-3p, miR-519c-3p mimic and corresponding control (miR-con) were bought from GeneChem (Shanghai, China). Tu suppress MAGI2-AS3 expression, and small interfering RNA si-MAGI2-AS3 and siR-con were purchased at GeneChem. Cells were seeded into 6-well plate and transfected with Lipo 2000 (Invitrogen) according to the manufacturer's instructions

2.4. RNA Extraction and Real-Time Quantitative PCR (RTqPCR) Assay. To isolate RNA from tissues and cells, TRIzol reagent (Invitrogen) was used. Afterward, RNA sample extracted was reverse transcribed into complementary DNA using TIANScript Kit (Tiangen, Beijing, China). RT-qPCR was conducted on BAI 7500 (Applied Biosystems, Foster City, CA, USA) using SYBR Green (Takara, Dalian, China). Primers used were synthesized by GeneChem and listed as follows: MAGI2-AS3: forward, $5^{\prime}$-CACCTTGCTTGACAACTTGA-3' ${ }^{\prime}$, reverse, 5' $^{\prime}$ CATTACAGCTCGGCTACTGC-3'; TXNIP: forward, 5' -CGCCTCCTGCTTGAAACTAAC-3', reverse, 5'-AATATACGCCGCTGGTTACACT-3';
miR-519c-3p: forward, $5^{\prime}$-GGCGGGAAAGTGCATCTTTTT-3', reverse, 5' - GTCGTATCCAGTGCAGGGTCCGAGGTATTCGCACTGGATACGACATCCTC-3'; GAPDH: forward, 5'-CGCTCTCTGCTCCTCCTGTTC-3, reverse, $5^{\prime}$-ATCCGTTGACTCCGACCTTCAC-3'; and U6 snRNA: forward, $5^{\prime}$-CTCGCTTCGGCAGCACA-3' ${ }^{\prime}$, reverse, $5^{\prime}$-AACGCTTCACGAATTTGCGT-3'. Expression levels were calculated with the $2-\Delta \Delta$ CT method with GAPDH or U6 snRNA as endogenous control.

2.5. Cell Proliferation Analysis. Cell Counting Kit-8 (CCK-8; Beyotime, Haimen, China) assay was conducted to measure cell proliferation. Cells were seeded in 96-well plate and measured every $24 \mathrm{~h}$ using a microplate reader after the addition of CCK-8 reagent for 4 times. Optical density was set as $450 \mathrm{~nm}$.

2.6. Cell Apoptosis Assay. Annexin V-FITC and PI were used to stain HCC cells based on manufacturer's instructions. Then, cell apoptosis rate including early to late apoptosis was analyzed at FACScan flow cytometry (BD Biosciences).

2.7. Bioinformatics Analysis. We explored the possible targets for MAGI2-AS3 using ENCORI and revealed miR-519c$3 p$ was a target. In addition, TargetScan was used to explore the targets for miR-519c-3p and identified YXNIP as a possible target.

Luciferase reporter assay: sequences of MAGI2-AS3 and TXNIP containing the binding region for miR-519c-3p were inserted into pmirGLO (Promega, Madison, WI, USA) to obtain MAGI2-AS3-WT and TXNIP-WT. The site-directed mutagenesis kit (Takara) was used to generate mutant luciferase vectors: MAGI2-AS3-MT and TXNIP-MT. Cells were transfected with luciferase vectors and miRNAs. Luciferase activity was measured after $48 \mathrm{~h}$ transfection using the dual-luciferase reporter kit (Promega).

2.8. RNA Pull-Down Assay. HCC cell lysates were incubated with the biotin labeled miR-519c-3p-WT or MT at room temperature for $2 \mathrm{~h}$. Then, streptavidin-labeled magnetic beads (Invitrogen) were added and incubated for $4 \mathrm{~h}$. After washed with lysis buffer, RNA was extracted and subjected to RT-qPCR analysis.

2.9. RNA Immunoprecipitation (RIP) Assay. RIP assay was performed using the Magna RI RIP Kit (Millipore, Billerica, MA, USA). Briefly, cells were lysed and incubated with antiAgo 2 or anti-IgG antibody at $4^{\circ} \mathrm{C}$ for $2 \mathrm{~h}$. Then, samples were mixed with magnetic beads at $4^{\circ} \mathrm{C}$ for overnight. Finally, beads were washed with RIP buffer and treated with TRIzol to isolate RNA sample and then subjected to RT-qPCR analysis.

2.10. Detection of MAGI2-AS3, miR-519c-3p, and TXNIP Expression at Public Access Tool. The expression levels of MAGI2-AS3, miR-519c-3p, and TXNIP in HCC tissues and 
normal tissues were analyzed at ENCORI website. Correction of MAGI2-AS3 with miR-519c-3p or TXNIP was also analyzed at ENCORI.

2.11. Xenograft Model. Study protocol was approved by Affiliated Hospital of Youjiang Medical University for Nationalities. BALB/c nude mice were injected with HCC cells with or without MAGI2-AS3 overexpression. Tumor width and length were measured every 7 days to calculate tumor volume with the formula: (width ${ }^{2} \times$ length)/2. 4 weeks after injection; mice were killed to obtain tumor tissues and then weighted to record tumor weight.

2.12. Statistical Analysis. Data were analyzed with SPSS (IBM, Armonk, NY, USA) and presented as mean \pm SD. Differences were analyzed with Student's $t$-test and one-way ANOVA. Expression correlations were analyzed using Pearson's correlation method. $P<0.05$ was considered as statistically significant.

\section{Results}

3.1. MAGI2-AS3 Was Decreased Expression in HCC. At first, RT-qPCR analysis showed MAGI2-AS3 expression was remarkedly decreased in HCC tissues compared with adjacent normal tissues (Figure 1(a)). In addition, we analyzed the MAGI2-AS3 in ENCORI and found the MAGI2-AS3 level was also decreased in tumor tissues compared with normal tissues (Figure 1(b)). Moreover, as expected, lower MAGI2-AS3 expression was observed in HCC cells compared with normal cell (Figure 1(c)). Hep3B with the lowest MAGI2-AS3 levels among the investigated HCC cells was selected for following analyses.

3.2. MAGI2-AS3 Inhibits HCC Cell Proliferation In Vitro. To explore the roles of MAGI2-AS3 on HCC, we performed gain and loss-of-function experiments on HCC cells. Transfection efficiency was validated by RT-qPCR (Figure 2(a)). CCK-8 assay revealed that MAGI2-AS3 overexpression remarkedly suppresses, while MAGI2-AS3 knockdown significantly stimulates cell proliferation in HCC cells compared with the negative controls (Figure 2(b)). Furthermore, the results of flow cytometry showed the cell apoptosis rate in the MAGI2-AS3 overexpressed group is higher than that in the pcDNA3.1 group (Figure 2(c)). Meanwhile, MAGI2-AS3 knockdown significantly repressed cell apoptosis compared with siR-con (Figure 2(c)).

3.3. MAGI2-AS3 Suppresses HCC Tumorigenesis In Vivo. Subsequently, we tested the roles of MAGI2-AS3 on HCC tumor growth in vivo. As displayed in Figure 3(a) and 3(b), tumors volume obtained from MAGI2-AS3 overexpressed groups was lower than those from control groups. Consistently, tumor weight was significantly reduced by MAGI2AS3 overexpression (Figure 3(c)). Then, we detected MAGI2-AS3 expression levels and showed MAGI2-AS3 was significantly elevated by pMAGI2-AS3 in tumors (Figure 3(d)).

3.4. $m i R-519 c-3 p$ Was a Target for MAGI2-AS3. To explore the miRNA target of MAGI2-AS3 in HCC, ENCORI was performed, and the prediction results showed MAGI2-AS3 interacts with miR-519c-3p (Figure 4(a)). Luciferase assay showed miR-519c-3p mimic can decrease luciferase activity of cells transfected with MAGI2-AS3-WT but not MAGI2AS3-MT (Figure 4(b)). Additionally, the RIP assay showed MAGI2-AS3 and miR-519c-3p were coenriched (Figure 4(c)). Moreover, we detected miR-519c-3p levels in HCC and showed miR-519c-3p expression was higher in HCC tissues and cells (Figures 4(d)-4(f)). Furthermore, we showed MAGI2-AS3 has a negative correction relationship with miR-519c-3p in HCC tissues (Figure 4(g)).

3.5. MAGI2-AS3 Regulates HCC Cell Proliferation by Regulating $m i R-519 c-3 p$. To explore whether the roles of MAGI2AS3 are mediated by miR-519c-3p, we transfected miR$519 c-3 p$ mimic into the MAGI2-AS3 overexpressed HCC cells. RT-qPCR showed levels of the miR-519c-3p in pMAGI2-AS3 transfected group were significantly lower than the cotransfection of the pMAGI2-AS3 and miR-519c$3 \mathrm{p}$ mimic group or pcDNA3.1 group (Figure 5(a)). CCK-8 assay, transwell invasion assay, and flow cytometry assay showed cotransfection of miR-519c-3p mimic increased cell proliferation and invasion and decreased cell apoptosis compared with the pMAGI2-AS3 group (Figures 5(b) and $5(c))$.

3.6. MAGI2-AS3 Regulates TXNIP Expression via Sponging $m i R-519 c-3 p$. Next, TargetScan revealed TXNIP was a potential target of miR-519c-3p (Figure 6(a)). Luciferase reporter assay further demonstrated that miR-519c-3p overexpression decreased luciferase activity of TXNIP-WT construct, while it did not affect the activity of cells transfected with TXNIP-MT construct (Figure 6(b)). RIP and RNA pull-down assay revealed that MAGI2-AS3 and TXNIP could interact with miR-519c-3p (Figures 6(c) and 6(d)). RT-qPCR showed TXNIP was increased expression in HCC tissues and cells (Figures 6(e)-6(g)). Pearson's correlation method showed MAGI2-AS3 was positively correlated with TXNIP (Figure 6(h)).

3.7. MAGI2-AS3 Exerts Its Function by Regulating TXNIP in HCC. Based on these observations, we hypothesized that MAGI2-AS3 may affect HCC progression via miR-519c-3p/ TXNIP axis. We then overexpressed TXNIP in miR-519c-3p overexpressed in HCC cells. As shown in Figure 7(a), the TXNIP level in the pTXNIP group was significantly higher than that in the pcDNA3.1 or pTXNIP and miR-519c-3p mimic group. CCK- 8 assay indicated that overexpression of TXNIP reversed the effects of cell proliferation induced by miR-519c-3p mimic (Figure 7(b)). Moreover, overexpression of TXNIP attenuated the effects of miR-519c-3p mimic on cell apoptosis (Figure $7(\mathrm{c})$ ). 

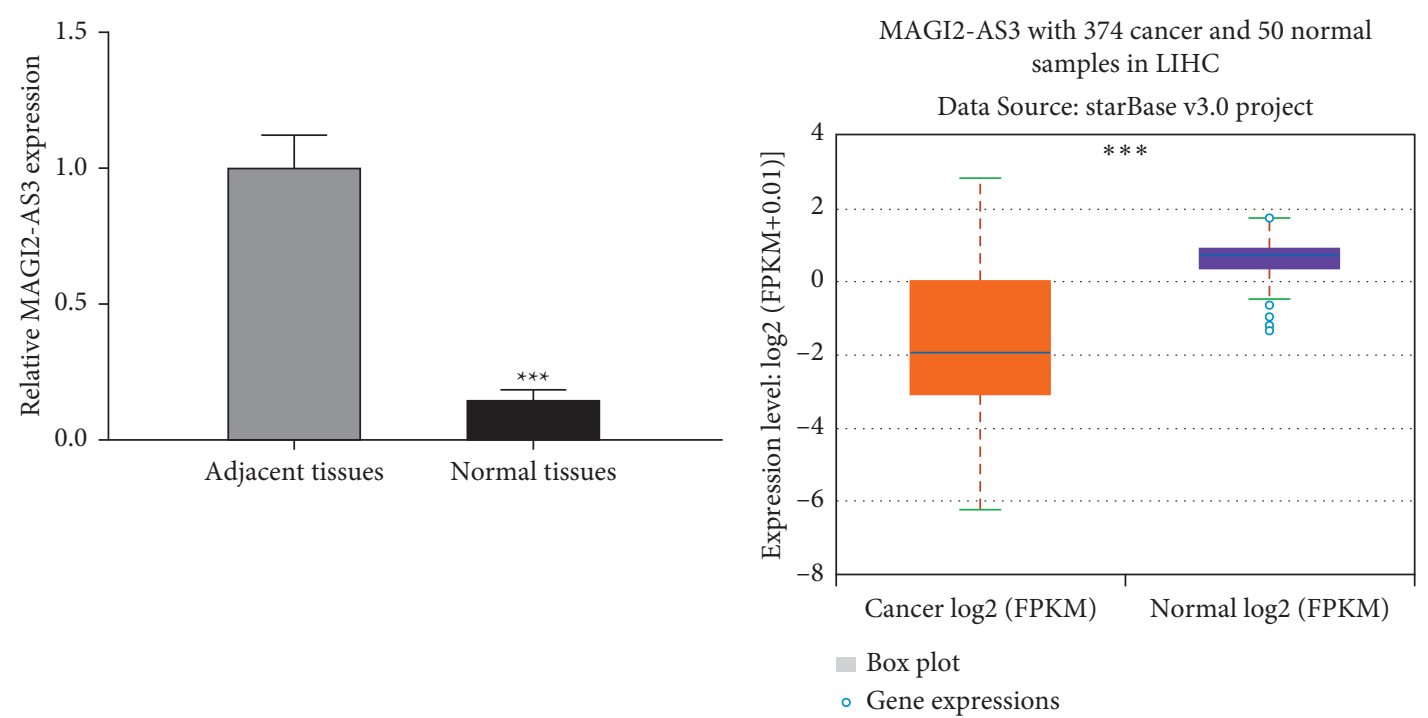

(a)

(b)

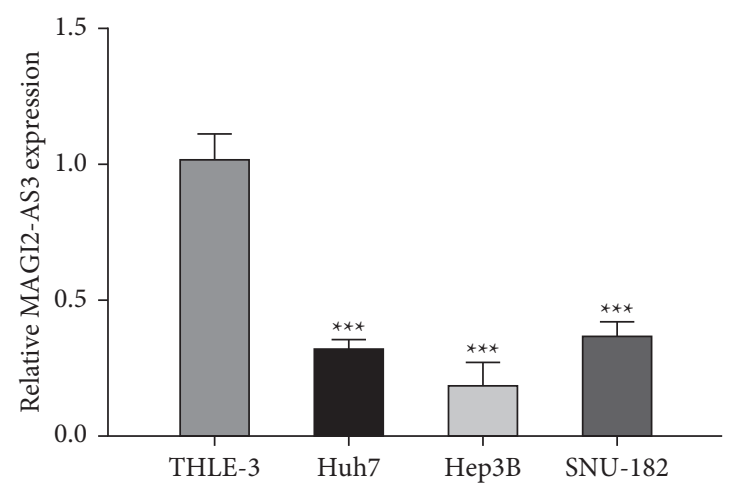

(c)

Figure 1: MAGI2-AS3 expression in HCC tissues and cell lines. (a) MAGI2-AS3 expression in HCC tissues and normal tissues analyzed by RT-qPCR. (b) MAGI2-AS3 expression in HCC tissues and normal tissues analyzed at ENCORI. (c) MAGI2-AS3 expression in HCC cells and normal cells analyzed by RT-qPCR. HCC, hepatocellular carcinoma; MAGI2-AS3, MAGI2 antisense RNA 3; RT-qPCR, real-time quantitative PCR.

\section{Discussion}

Emerging evidence has indicated that lncRNAs are crucial mediators for the progression of HCC. For example, lncRNA taurine upregulated gene 1 was found elevated expression in HCC and promotes tumor growth and metastasis by promoting distal-less homeobox 2 expression via sponging miR216b-5p [10]. IncRNA DLGAP1-AS1 revealed upregulated expression in HCC and promoted tumorigenesis through activating the $\mathrm{Wnt} / \beta$-catenin pathway [11]. In this study, we revealed that MAGI2-AS3 was decreased expression in HCC by analyzing tissue samples, cells, and online database. Moreover, we revealed that MAGI2-AS3 could inhibit HCC cell proliferation in vitro using gain and loss-of-function experiments. In addition, we showed that MAGI2-AS3 could inhibit HCC tumor growth in vivo through gain-of-function experiment. These results indicated that MAGI2-AS3 may function as tumor suppressive role in HCC.

To date, the numbers of identified lncRNAs were gradually increasing, but the mechanisms related to their roles in carcinogenesis are largely elusive. Hence, we aimed to discover the potential mechanisms of MAGI2-AS3 in HCC using the ceRNA theory. miR-3163, miR-141, and miR-200a were previously identified as miRNA targets for MAGI2-AS3 in cancers [7, 12]. Here, we predicted and validated that miR-519c-3p was a possible target for MAGI2AS3 using bioinformatic analysis, luciferase activity assay, and RIP assay. After that, we searched previous literature regarding the roles of $\mathrm{miR}-519 \mathrm{c}-3 \mathrm{p}$ in cancer and found miR-519c-3p is a newly identified miRNA with very little studies to report its functions. Therefore, considering the roles of miR-519c-3p in cancer remain largely unknown, we explored whether miR-519c-3p was a functional target for MAGI2-AS3. Our study demonstrated that the overexpression of miR-519c-3p could partially overwrite the functions of MAGI2-AS3 in HCC. These results suggested a tumor promoting role of miR-519-3p in HCC, which is in consistent with the previous study [9]. Taken together, our study combined with the previous study validated the importance of miR-519c-3p in HCC. 

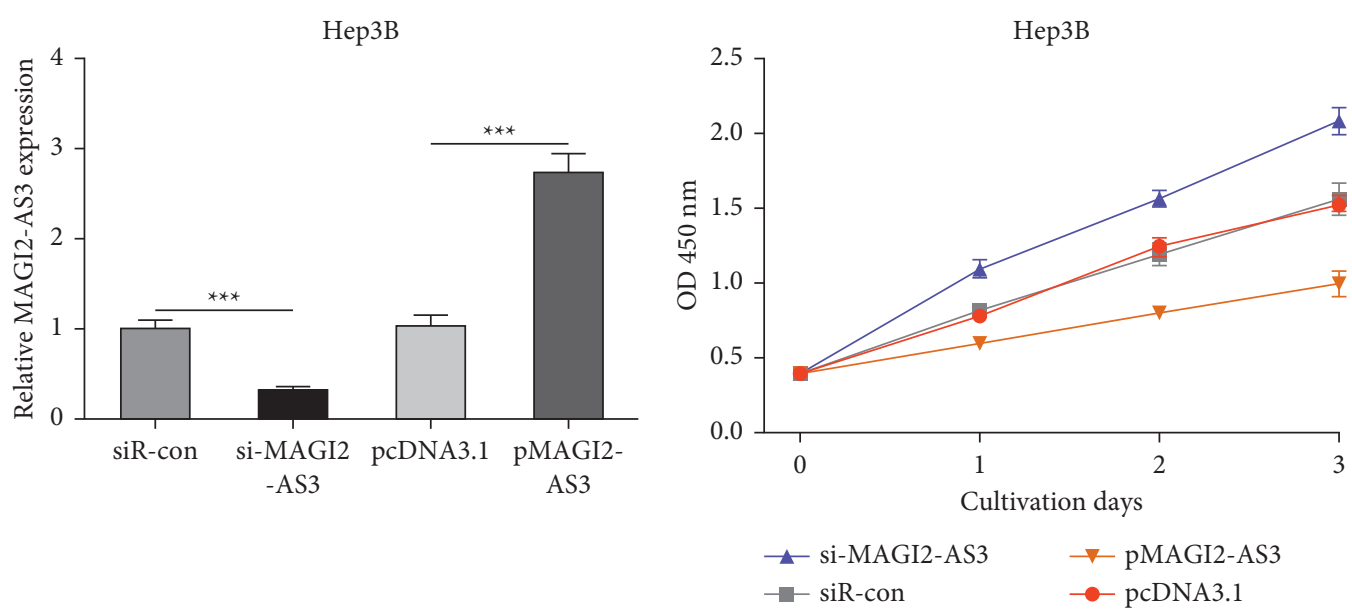

(a)

(b)
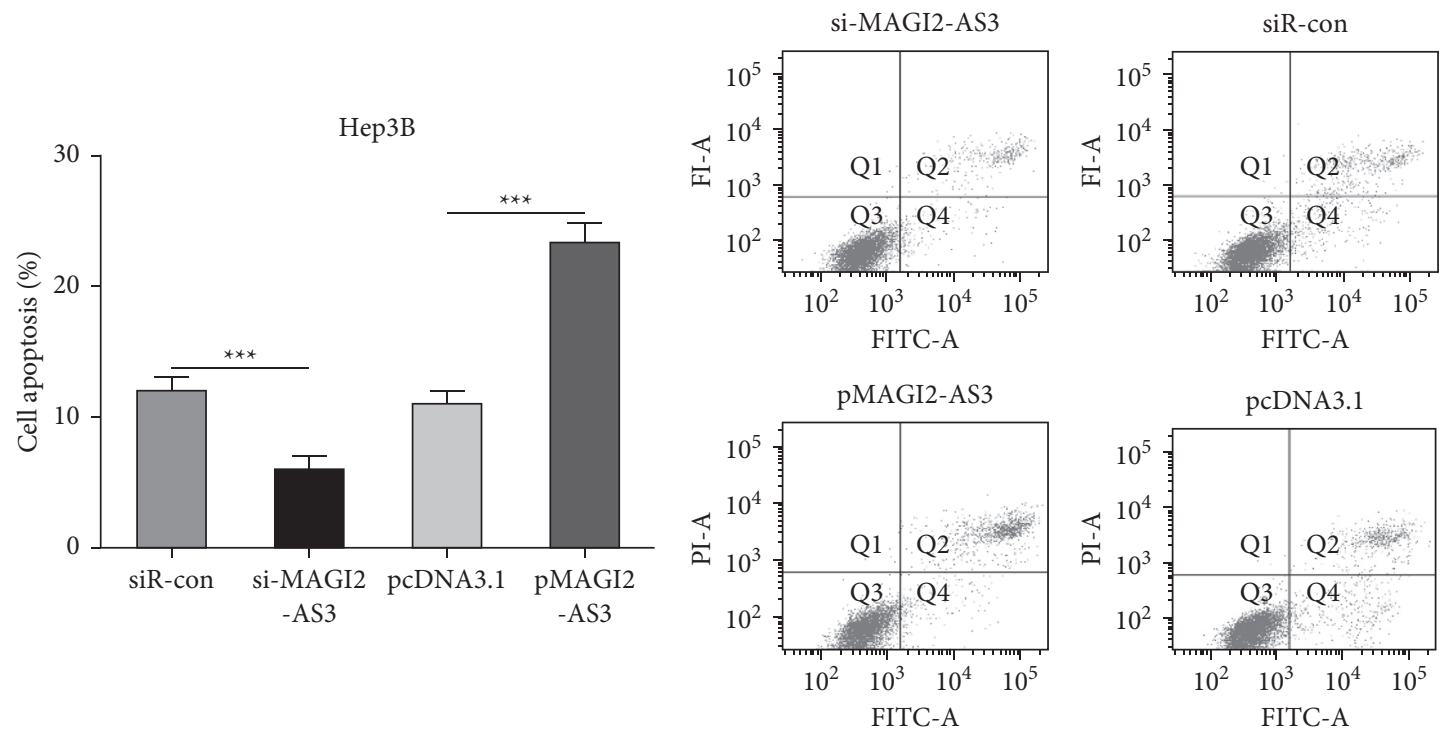

(c)

FIGURE 2: MAGI2-AS3 regulating HCC tumor progression in vitro. (a) MAGI2-AS3 expression in HCC cells with pMAGI2-AS3 or si-MAGI2-AS3 transfection. (b) Cell viability of HCC cells with pMAGI2-AS3 or si-MAGI2-AS3 transfection. (c) Cell apoptosis rate of HCC cells with pMAGI2-AS3 or si-MAGI2-AS3 transfection. HCC, hepatocellular carcinoma; MAGI2-AS3, MAGI2 antisense RNA 3; RT-qPCR, real-time quantitative PCR; si-MAGI2-AS3, small interfering RNA against MAGI2-AS3; siR-con, negative control siRNA.

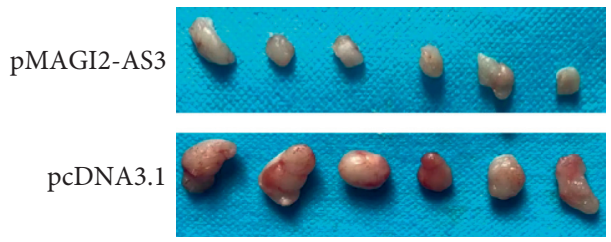

(a)

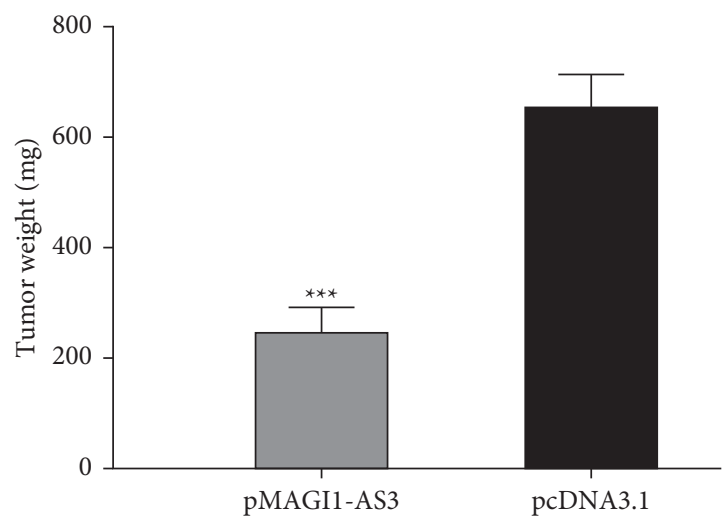

(b)

Figure 3: Continued. 


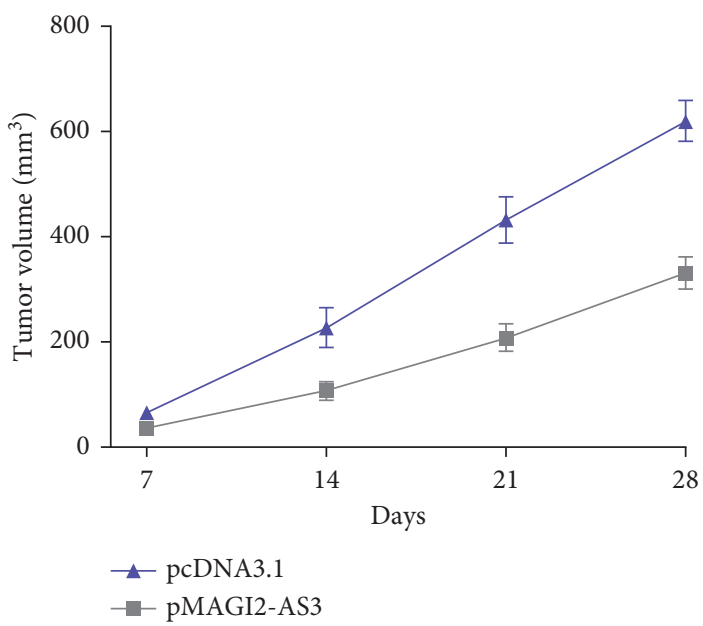

(c)

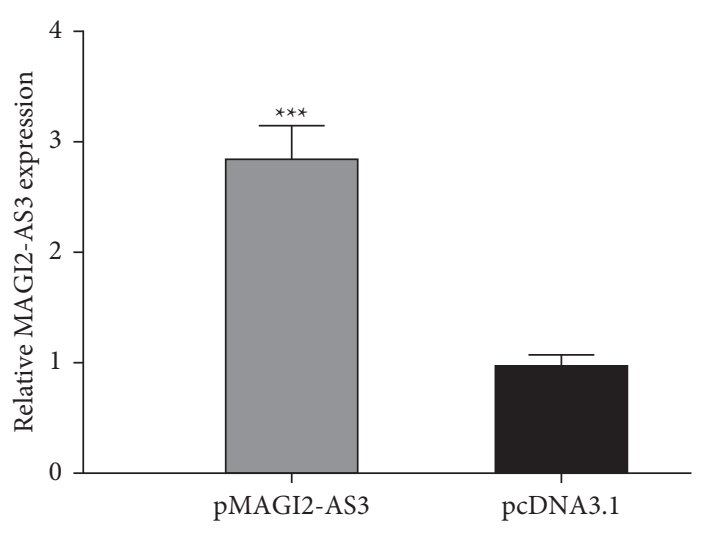

(d)

Figure 3: MAGI2-AS3 regulating HCC tumor progression in vivo. (a) Images of the tumor tissues transfected with pMAGI2-AS3 or pcNDA3.1. (b) Tumor size of the tumor tissues transfected with pMAGI2-AS3 or pcNDA3.1. (c) Tumor weight of the tumor tissues transfected with pMAGI2-AS3 or pcNDA3.1. (d) MAGI2-AS3 expression in tumor tissues transfected with pMAGI2-AS3 or pcNDA3.1. HCC, hepatocellular carcinoma; MAGI2-AS3, MAGI2 antisense RNA 3; RT-qPCR, real-time quantitative PCR.

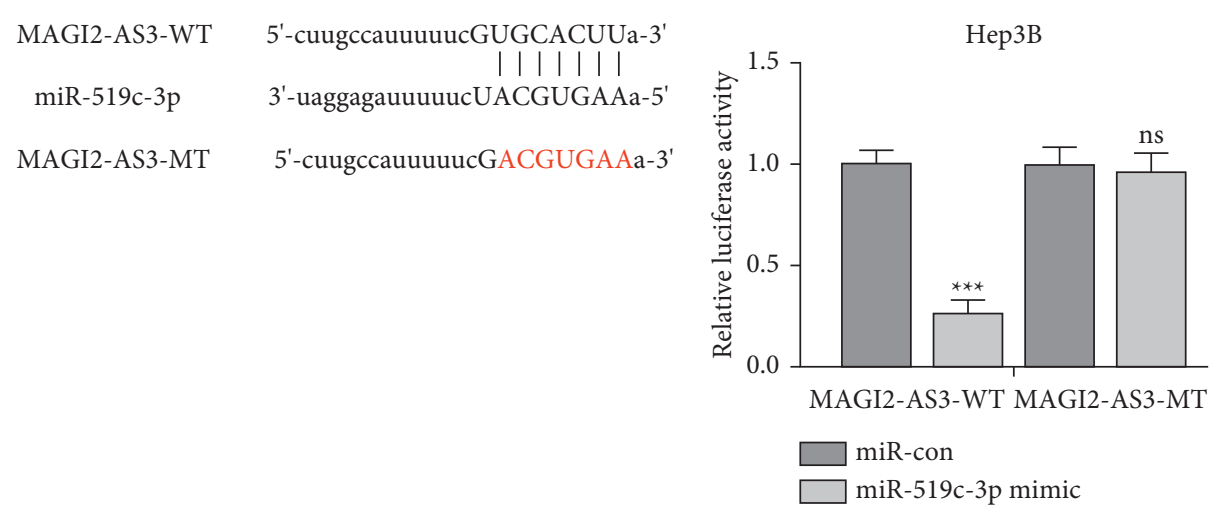

(a)

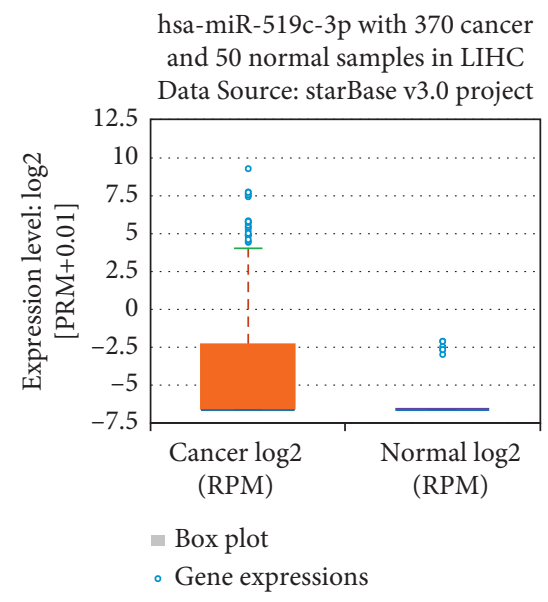

(d)
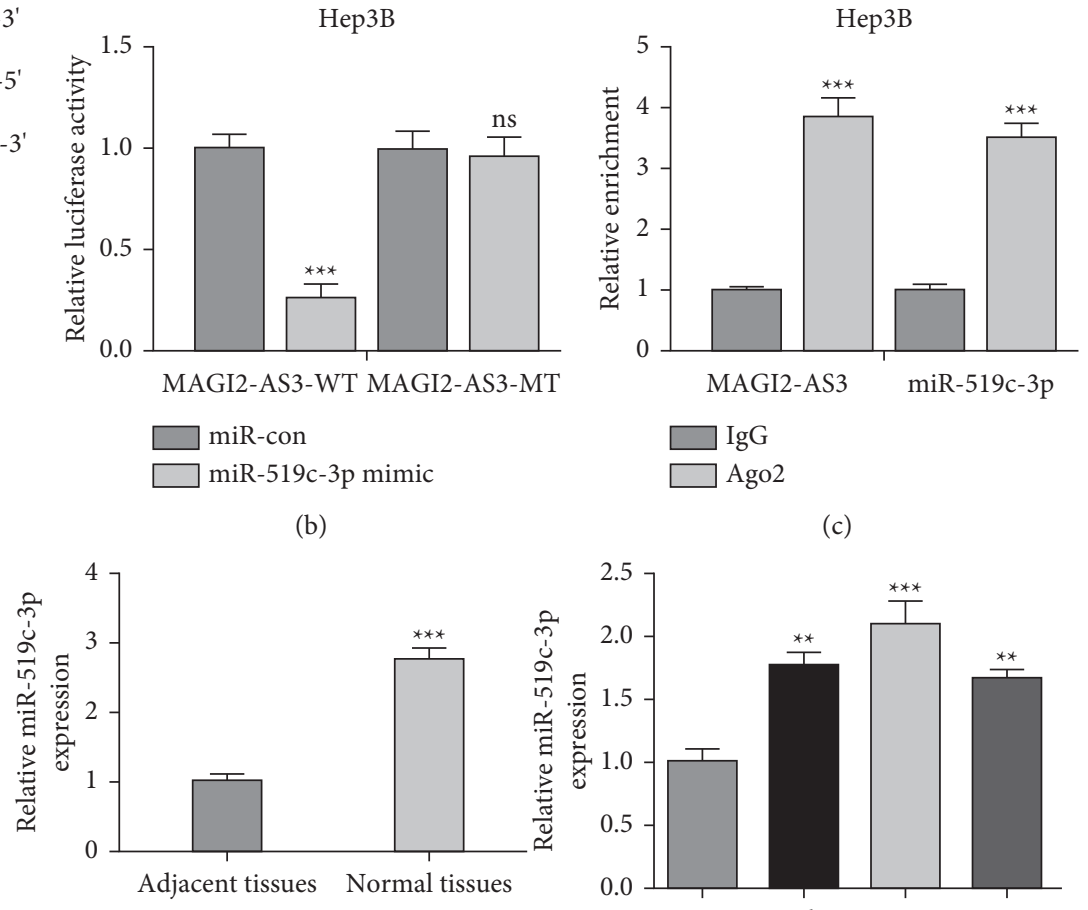

(c)

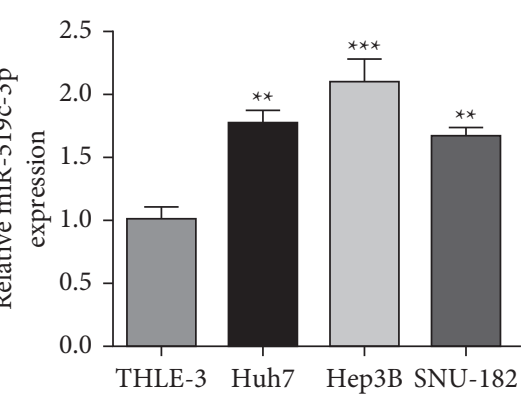

(e)

Figure 4: Continued. 


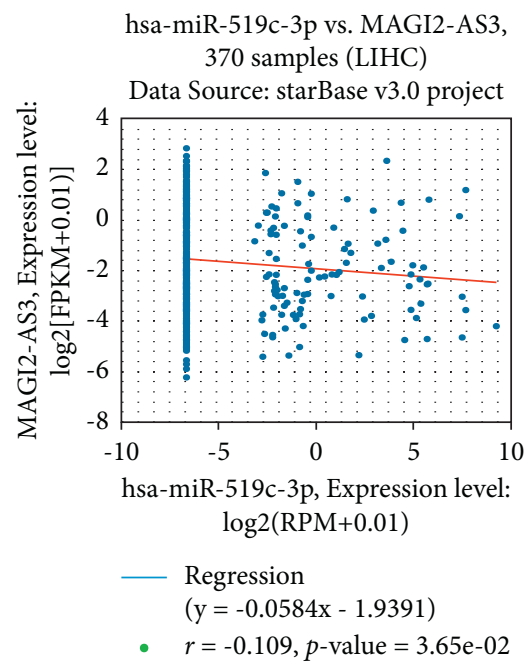

(g)

FIGURE 4: MAGI2-AS3 directly interacts with miR-519c-3p. (a) Binding region between MAGI2-AS3 and miR-519c-3p predicted by ENCORI. (b) Luciferase assay showing miR-519c-3p mimic decreased luciferase activity of MAGI2-AS3-WT but not MAGI2-AS3-MT. (c) RIP assay showing the coenrichment of MAGI2-AS3 and miR-519c-3p. (d) MAGI2-AS3 expression in HCC tissues and normal tissues analyzed at ENCORI. (e) MAGI2-AS3 expression in HCC tissues and normal tissues analyzed by RT-qPCR. (f) MAGI2-AS3 expression in HCC cells and normal cells analyzed by RT-qPCR. (g) Correlation of MAGI2-AS3 and miR-519c-3p analyzed at ENCORI. HCC, hepatocellular carcinoma; MAGI2-AS3, MAGI2 antisense RNA 3; RT-qPCR, real-time quantitative PCR; WT, wild type; MT, mutant; RIP, RNA immunoprecipitation; miR-519c-3p, microRNA-519c-3p; miR-con, negative control miRNA.

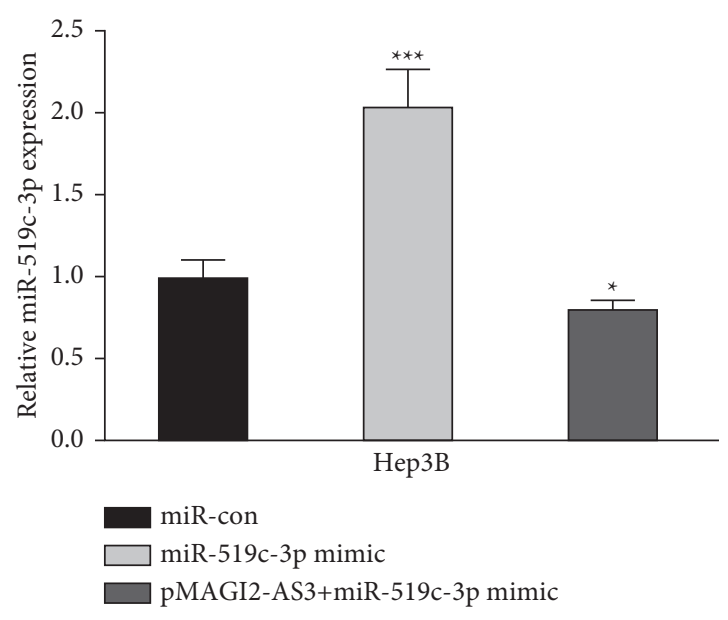

(a)

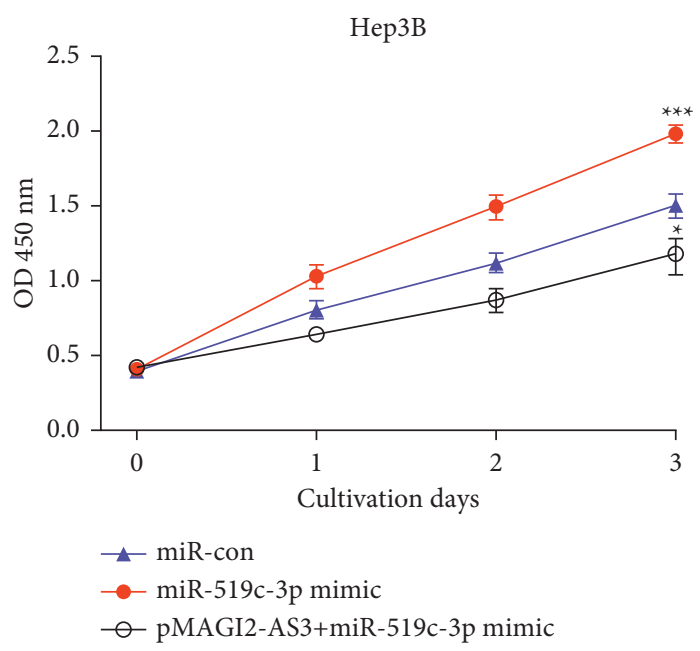

(b)

FIgURE 5: Continued. 


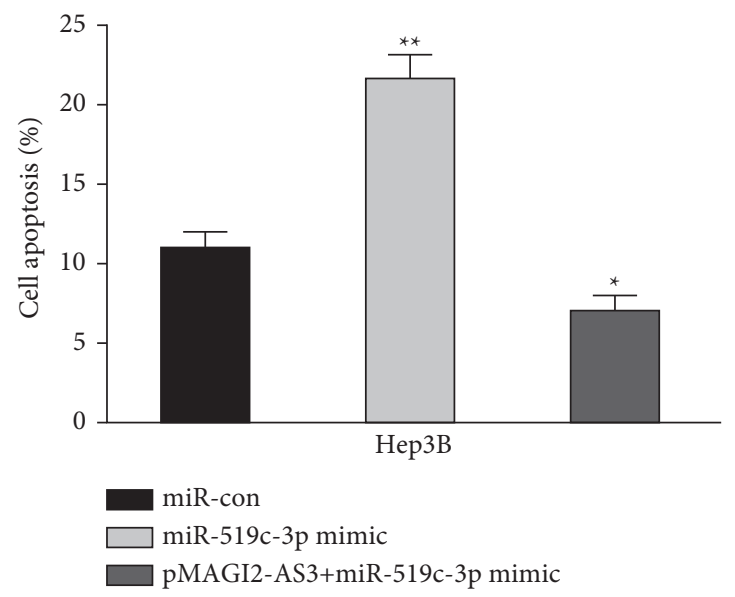

(c)

FIGURE 5: miR-519c-3p mimic restores the effects of MAGI2-AS3 on HCC cells. (a) MAGI2-AS3 expression in HCC cells with pMAGI2AS3+miR-519c-3p mimic, miR-519c-3p mimic, or miR-con transfection. (b) Cell viability of HCC cells with pMAGI2-AS3+miR-519c-3p mimic, miR-519c-3p mimic, or miR-con transfection. (c) Cell apoptosis rate of HCC cells with pMAGI2-AS3+miR-519c-3p mimic, miR519c-3p mimic, or miR-con transfection. HCC, hepatocellular carcinoma; MAGI2-AS3, MAGI2 antisense RNA 3; RT-qPCR, real-time quantitative PCR; miR-519c-3p, microRNA-519c-3p; miR-con, negative control miRNA.

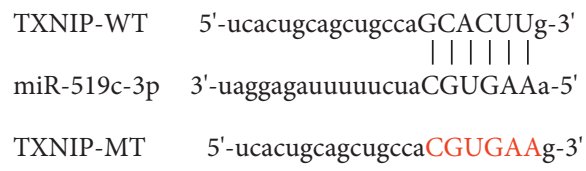

(a)

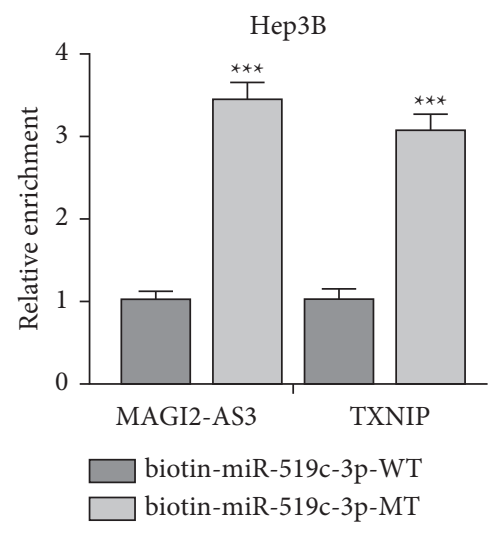

(d)

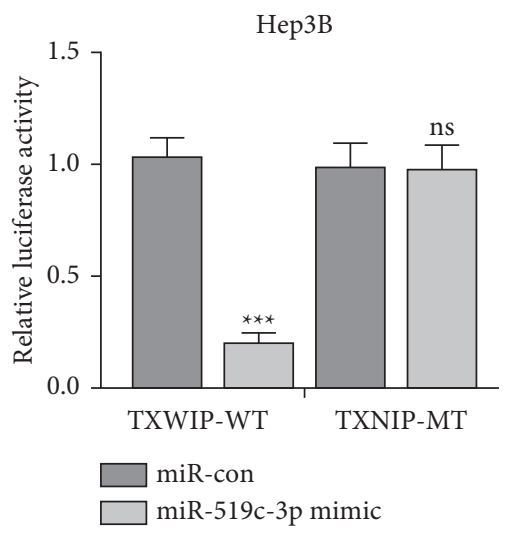

(b)

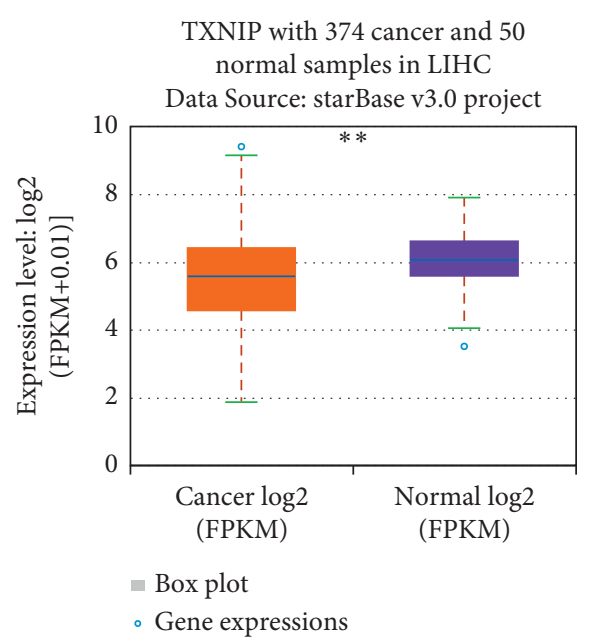

(e)

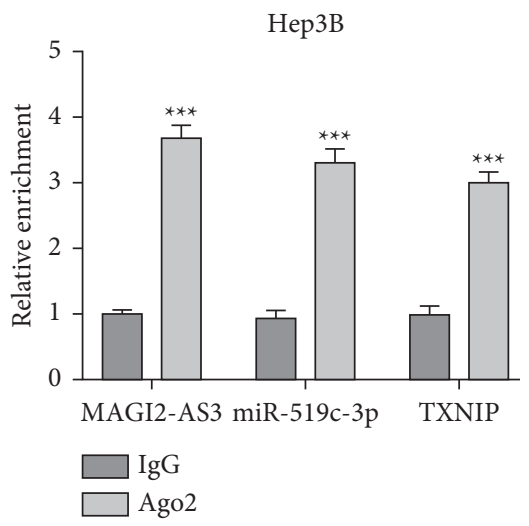

(c)

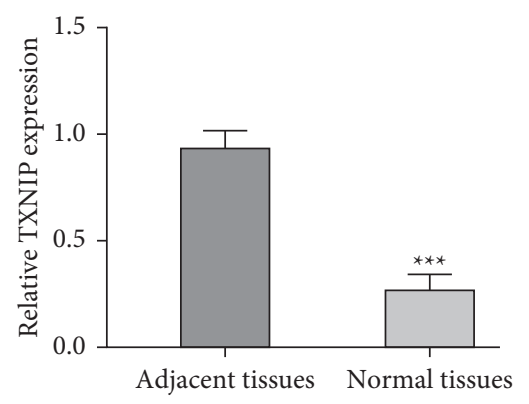

(f)

FIgURE 6: Continued. 


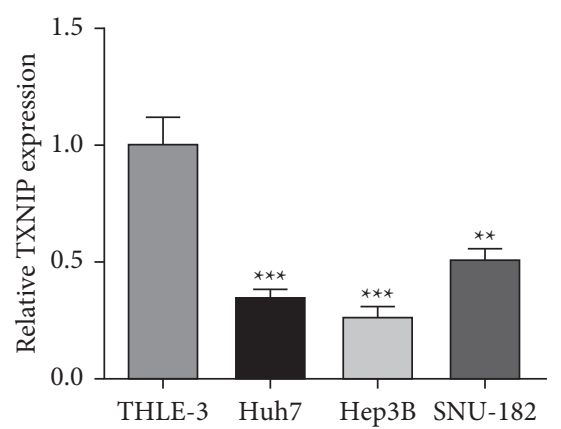

(g)

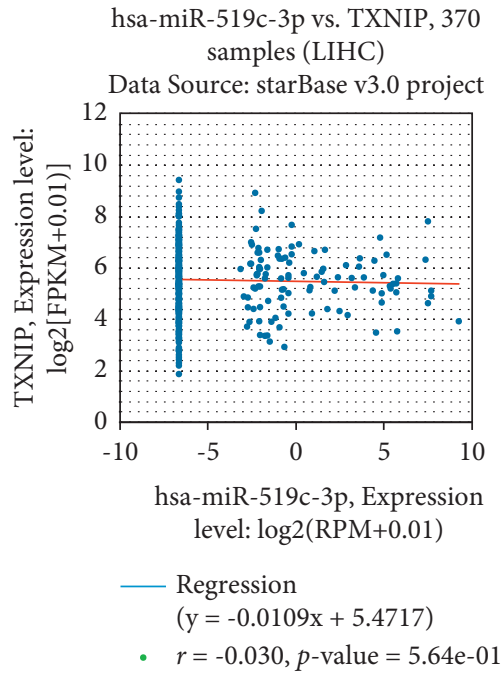

(h)

FIgURE 6: TXNIP was direct target of miR-519c-3p. (a) Binding region between TXNIP and miR-519c-3p predicted by TargetScan. (b) Luciferase assay showing miR-519c-3p mimic decreased luciferase activity of TXNIP-WT but not TXNIP-MT. (c) RIP assay showed the coenrichment of MAGI2-AS3, TXNIP, and miR-519c-3p. (d) MAGI2-AS3 and TXNIP expression in complex pulled down by biotin-miR519c-3p-WT or biotin-miR-519c-3p-MT. (e) MAGI2-AS3 expression in HCC tissues and normal tissues analyzed at ENCORI. (f) MAGI2AS3 expression in HCC tissues and normal tissues analyzed by RT-qPCR. (g) MAGI2-AS3 expression in HCC cells and normal cells analyzed by RT-qPCR. (h) Correlation of MAGI2-AS3 and miR-519c-3p analyzed at ENCORI. HCC, hepatocellular carcinoma; MAGI2AS3, MAGI2 antisense RNA 3; RT-qPCR, real-time quantitative PCR; WT, wild type; MT, mutant; RIP, RNA immunoprecipitation; miR519c-3p, microRNA-519c-3p; miR-con, negative control miRNA; TXNIP, thioredoxin interacting protein.

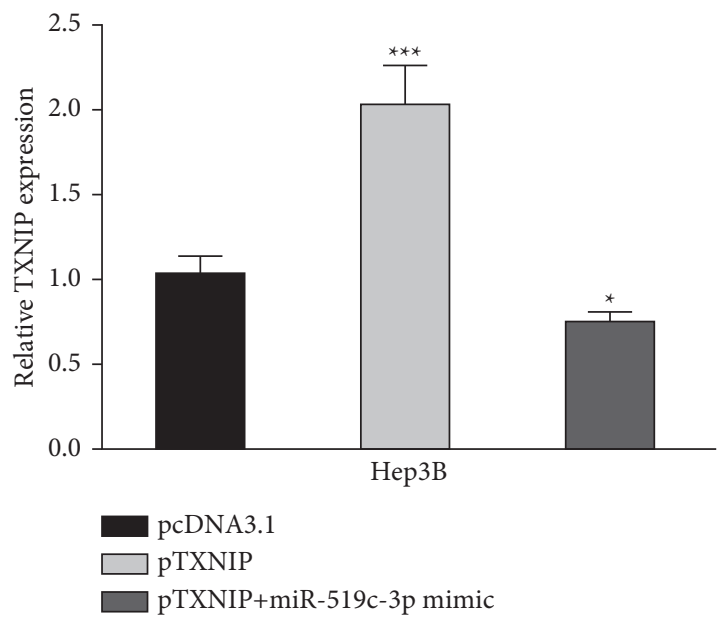

(a)

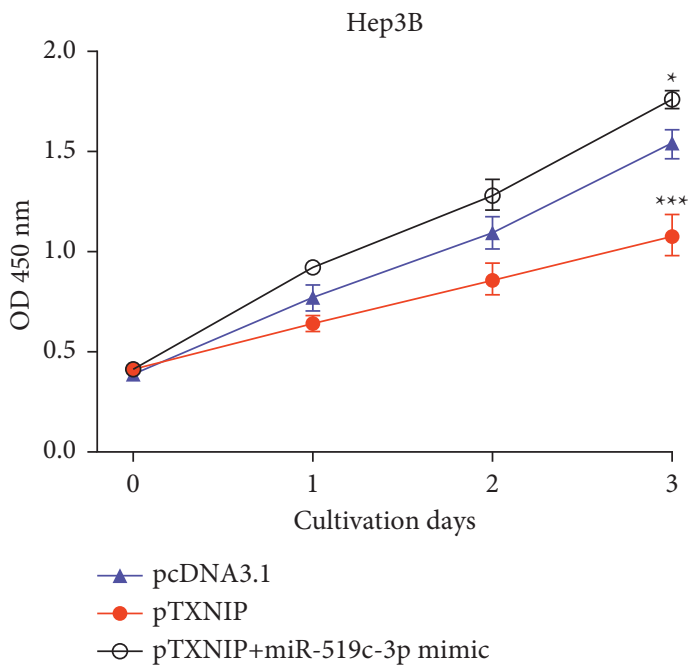

(b)

Figure 7: Continued. 


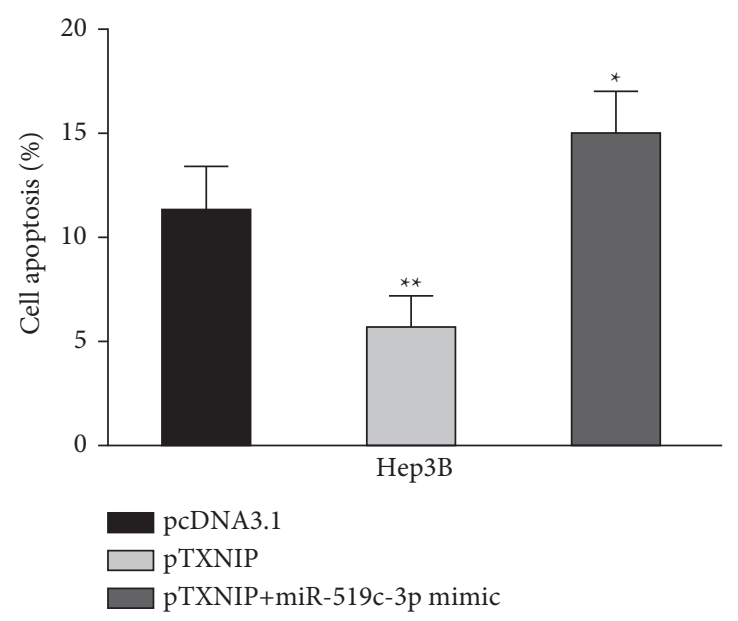

(c)

FIgURe 7: MAGI2-AS3 affects HCC behaviors through regulating TXNIP. (a) TXNIP expression in HCC cells with pTXNIP + miR-519c-3p mimic, pTXNIP, or pcDNA3.1 transfection. (b) Cell viability of HCC cells with pTXNIP + miR-519c-3p mimic, pTXNIP, or pcDNA3.1 transfection. (c) Cell apoptosis rate of HCC cells with pTXNIP + miR-519c-3p mimic, pTXNIP, or pcDNA3.1 transfection. HCC, hepatocellular carcinoma; MAGI2-AS3, MAGI2 antisense RNA 3; RT-qPCR, real-time quantitative PCR; miR-519c-3p, microRNA-519c-3p; miR-con, negative control miRNA; TXNIP, thioredoxin interacting protein.

TXNIP, a protein induced by vitamin D3, is originally named as vitamin D3 upregulated protein 1 [13]. TXNIP was revealed decreased expression in renal cell carcinoma, and its low expression was associated with advance tumor stages and poorer overall survival [14]. In addition, TXNIP expression revealed could be induced by MondoA and to inhibit cervical cancer cell proliferation, migration, and invasion [15]. In our study, we discovered that TXNIP was a target for miR-519c$3 p$ and MAGI2-AS3 that regulates TXNIP expression via sponging miR-519c-3p. Rescue experiments further confirmed that MAGI2-AS3 regulates HCC progression via the miR-519c-3p/TXNIP axis. A very recent study performed single-cell RNA-seq of colorectal cancer samples and showed the low activity of the MondoA-TXNIP axis is associated with the intratumoral Tregs content [16]. Also, it should be noted that suppressing the activity of MondoA-TXNIP can facilitate Th17 inflammation and stimulate $\mathrm{CD}^{+} \mathrm{T}$ cell exhaustion, indicating the roles of TXNIP in regulating tumor microenvironment [16]. As we identified that TXNIP was a target of MAGI2-AS3 and results in the reduced expression in of TXNIP in HCC and impaired cancer progression, we suspected that altered MAGI2-AS3 expression may also cause changes in the immune cell populations in the microenvironment of HCC patients. It will be interesting to further analyze the different types and proportion of immune cells after knockdown of overexpressing MAGI2-AS3 in HCC to dig out the deep mechanisms by which MAGI2-AS3 affects HCC progression.

Our previous study highlighted that MAGI2-AS3 was an epigenetic regulator to regulate the overall $\mathrm{H} 3 \mathrm{~K} 4 \mathrm{me} 2$ demethylation level and affect the tumorigenesis and progression of HCC [6]. In this present study, we provide new evidence that MAGI2-AS3 could affect HCC progression via a new ceRNA mechanism, which strengthen our previous study and also new insights into the functions of MAGI2AS3 in HCC.

\section{Conclusion}

In summary, we revealed that MAGI2-AS3 was decreased expression in HCC, and it can inhibit HCC progression via the miR-519c-3p/TXNIP axis, which provided insights that MAGI2-AS3 may be the effective target for HCC treatment.

\section{Data Availability}

The data used to support the findings of this study are available from corresponding author upon request.

\section{Conflicts of Interest}

The authors declare that they have no conflicts of interest.

\section{Authors' Contributions}

Huamei Wei and Qianli Tang contributed equally to this work.

\section{Acknowledgments}

This work was supported by Special Funding for Guangxi Special Experts.

\section{References}

[1] J. D. Yang and L. R. Roberts, "Hepatocellular carcinoma: a global view," Nature Reviews Gastroenterology \& Hepatology, vol. 7, no. 8, pp. 448-458, 2010.

[2] D. J. Erstad and K. K. Tanabe, "Prognostic and therapeutic implications of microvascular invasion in hepatocellular carcinoma," Annals of Surgical Oncology, vol. 26, no. 5, pp. 1474-1493, 2019.

[3] A. Forner, M. Reig, and J. Bruix, "Hepatocellular carcinoma," The Lancet, vol. 391, no. 10127, pp. 1301-1314, 2018. 
[4] M. Guttman and J. L. Rinn, "Modular regulatory principles of large non-coding RNAs," Nature, vol. 482, no. 7385, pp. 339-346, 2012.

[5] T. Gutschner and S. Diederichs, "The hallmarks of cancer," RNA Biology, vol. 9, no. 6, pp. 703-719, 2012.

[6] J. Pu, J. Wang, H. Wei et al., "IncRNA MAGI2-AS3 prevents the development of HCC via recruiting KDM1A and promoting H3K4me2 demethylation of the RACGAP1 promoter," Molecular Therapy - Nucleic Acids, vol. 18, pp. 351-362, 2019.

[7] H. Ren, Z. Li, Z. Tang, J. Li, and X. Lang, "Long noncoding MAGI2-AS3 promotes colorectal cancer progression through regulating miR-3163/TMEM106B axis," Journal of Cellular Physiology, vol. 235, no. 5, pp. 4824-4833, 2020.

[8] X. Qi, D.-H. Zhang, N. Wu, J.-H. Xiao, X. Wang, and W. Ma, "ceRNA in cancer: possible functions and clinical implications," Journal of Medical Genetics, vol. 52, no. 10, pp. 710-718, 2015.

[9] L. Wang, H. Mo, Y. Jiang et al., "MicroRNA-519c-3p promotes tumor growth and metastasis of hepatocellular carcinoma by targeting BTG3," Biomedicine \& Pharmacotherapy, vol. 118, Article ID 109267, 2019.

[10] Q. Dai, J. Deng, J. Zhou et al., "Long non-coding RNA TUG1 promotes cell progression in hepatocellular carcinoma via regulating miR-216b-5p/DLX2 axis," Cancer Cell International, vol. 20, no. 8, p. 8, 2020.

[11] Y. Lin, Z. Jian, H. Jin et al., "Long non-coding RNA DLGAP1AS1 facilitates tumorigenesis and epithelial-mesenchymal transition in hepatocellular carcinoma via the feedback loop of miR-26a/b-5p/IL-6/JAK2/STAT3 and Wnt/ $\beta$-catenin pathway," Cell Death \& Disease, vol. 11, no. 1, p. 34, 2020.

[12] D. Li, J. Wang, M. Zhang et al., "LncRNA MAGI2-AS3 is regulated by $\mathrm{BRD} 4$ and promotes gastric cancer progression via maintaining ZEB1 overexpression by sponging miR-141/ 200a," Molecular Therapy-Nucleic Acids, vol. 19, pp. 109-123, 2019.

[13] K.-S. Chen and H. F. DeLuca, "Isolation and characterization of a novel cDNA from HL-60 cells treated with 1,25-dihydroxyvitamin D-3," Biochimica et Biophysica Acta (BBA) Gene Structure and Expression, vol. 1219, no. 1, pp. 26-32, 1994.

[14] Y. Gao, J. C. Qi, X. Li, J. P. Sun, H. Ji, and Q. H. Li, “Decreased expression of TXNIP predicts poor prognosis in patients with clear cell renal cell carcinoma," Oncology Letters, vol. 19, no. 1, pp. 763-770, 2020.

[15] J. Zhang, X. Tian, H. Yin et al., "TXNIP induced by MondoA, rather than ChREBP, suppresses cervical cancer cell proliferation, migration and invasion," Journal of Biochemistry, vol. 167, no. 4, pp. 371-377, 2002.

[16] Y. Lu, Y. Li, Q. Liu et al., "MondoA-TXNIP axis maintains regulatory $\mathrm{T}$ cell identity and function in colorectal cancer microenvironment," Gastroenterology, vol. 21, 2021. 